\title{
A giant submandibular sialolith - How to manage?
}

SADJ August 2020, Vol. 75 No. 7 p387 - p390

\author{
J Anand ${ }^{1}$, A Suresh ${ }^{2}$, AK Desai ${ }^{3}$
}

\section{ABSTRACT}

The presence of a sialolith is one of the most common diseases of salivary gland. It is relatively common in submandibular salivary glands and its duct.

This case report is of a patient who presented at our unit with a history of severe pain and swelling on floor of the mouth, which was clinically and radiographically diagnosed as a sialolith. The diagnostic and treatment protocol in managing a patient with a giant sialolith is enumerated in this manuscript.

\section{INTRODUCTION}

One of the most common diseases that affect the salivary glands and a major cause of salivary gland dysfunction is salivary stone. ${ }^{1}$

Studies have reported that the overall incidence is $1 \%$ in the population. ${ }^{2}$ Most of the cases remain asymptomatic.

The incidence of occurrence in sub-mandibular region is relatively high, $80-90 \%$ as compared to parotid region, $5-20 \%$ and the sub-lingual and other minor salivary glands which range from $0-10 \% .^{3,4,5}$

When minor salivary glands are involved mainly in the buccal mucosa, of the upper lip which forms a firm nodule which may mimic a tumour. The largest salivary gland stones are formed by sub-mandibular glands. Salivary stones are single in $70-80 \%$ of the cases and are also presented as multiple, usually two or three in number rarely.

Author affiliations:

1. Jawahar Anand: MDS, Assistant Professor, Department of Oral and Maxillofacial Surgery, SDM College of Dental Sciences, Shri Dharmasthala Manjunatheshwara University, Dharwad. ORCID Number: 0000-0001-6730-9609

2. Amal Suresh: MDS, Resident, Department of Oral and Maxillofacial Surgery, SDM College of Dental Sciences, Shri Dharmasthala Manjunatheshwara University, Dharwad ORCID Number: 0000-0002-9239-9930

3. Anil K Desai: MDS, Professor and Head of Department, Dept. Of Oral and maxillofacial surgery, SDM Craniofacial Surgery and Research Centre, SDM College of Dental Sciences and Hospital, Dharwad, Karnataka, India.

Corresponding author: Jawahar Anand

Asst Professor, Dept of Oral and Maxillofacial Surgery, SDM Craniofacial Surgery and Research Centre, SDM College of Dental Sciences, Dharwad.

Email: jawanand@gmail.com

Author contributions:

1. Jawahar Anand: Principal Researcher - 33.3\%

2. Amal Suresh: Writing the manuscript $-33.3 \%$

3. Anil K Desai: Mentor - $33.3 \%$
Nearly $88 \%$ of sialoliths are reported to be of a size less than $<10 \mathrm{~mm}$ in dimensions. According to literature the development of an atypically large, $>15 \mathrm{~mm}$ sialolith was found to be sporadic. ${ }^{6}$

Most common manifestations of salivary lithiasis include pain and swelling, especially during pre-meal period wherein the salivary flow is stimulated. The sialolith obstructs the normal salivary flow which can lead to multiple disabilities in the oral cavities.

Reports of huge salivary calculi have been reported in the literature. This manuscript is a case report of a Submandibular sialolith and describes the clinical presentation, examination, investigations, and the surgical excision.

\section{CASE REPORT}

A 69 years old male patient reported to our unit with chief complaint of pain in left side of floor of mouth for the past 15 days. He presented with pain associated with a diffuse swelling on the left side in the floor of mouth.

The pain was sudden in onset, with severity in intensity and continuous in nature. The pain was aggravated with eating. The associated swelling was initially minor and gradually increased to the present size over time.

On examination a solitary diffused swelling was noted in left side of floor of mouth with a sinus opening in relation to the 36,37 region (Figure $1 \mathrm{~A}$ ). Also, an extraoral swelling was noted in the sub-mandibular region measuring about $2 \mathrm{~cm}$ in diameter. Swelling was oval in shape, firm, tender and not attached to underlying structures. The left submandibular lymph node was palpable and was tender.

A mandibular occlusal radiograph was advised, which revealed a homogenous radio-opaque structure in left submandibular region in relation to the 36,37 area, which was oval in shape with well-defined borders and measuring approximately $1 \times 1 \mathrm{~cm}$ (Figure 2A).

Also, a CBCT was advised to determine the position of the sialolith (Figure 2 B, C, D). Based on the clinical and radiographic presentation we came to a diagnosis of a Surgical excision of the sialolith under local anaesthesia was suggested as the treatment plan.

Routine minor surgery protocol was followed and 2\% lignocaine with adrenaline 1:80,000 was infiltrated around 
the pathology. An incision was placed of around $1 \mathrm{~cm}$ over the most prominent part of the pathology. Blunt dissection was carried out through the Wharthon's and the sialolith was identified to expose it completely (Figure 1B). The sialolith was removed in toto which measured $2.2 \times 1.9$ centimetres and weighed 3.33 grams (Figure $1 \mathrm{C}$ and 3 ).

The duct was examined using Adson's forceps and mucous secretions were seen. The duct irrigation was done using betadine and saline. Milking of gland was performed to confirm the normal function of salivary gland. 5 simple interrupted 3-0 Black Braided Silk sutures were placed.

Postoperative instructions were given, to the patient and antibiotics and analgesics were prescribed for 5 days. The wound healed uneventfully without any complications. No recurrence was seen until the present date.
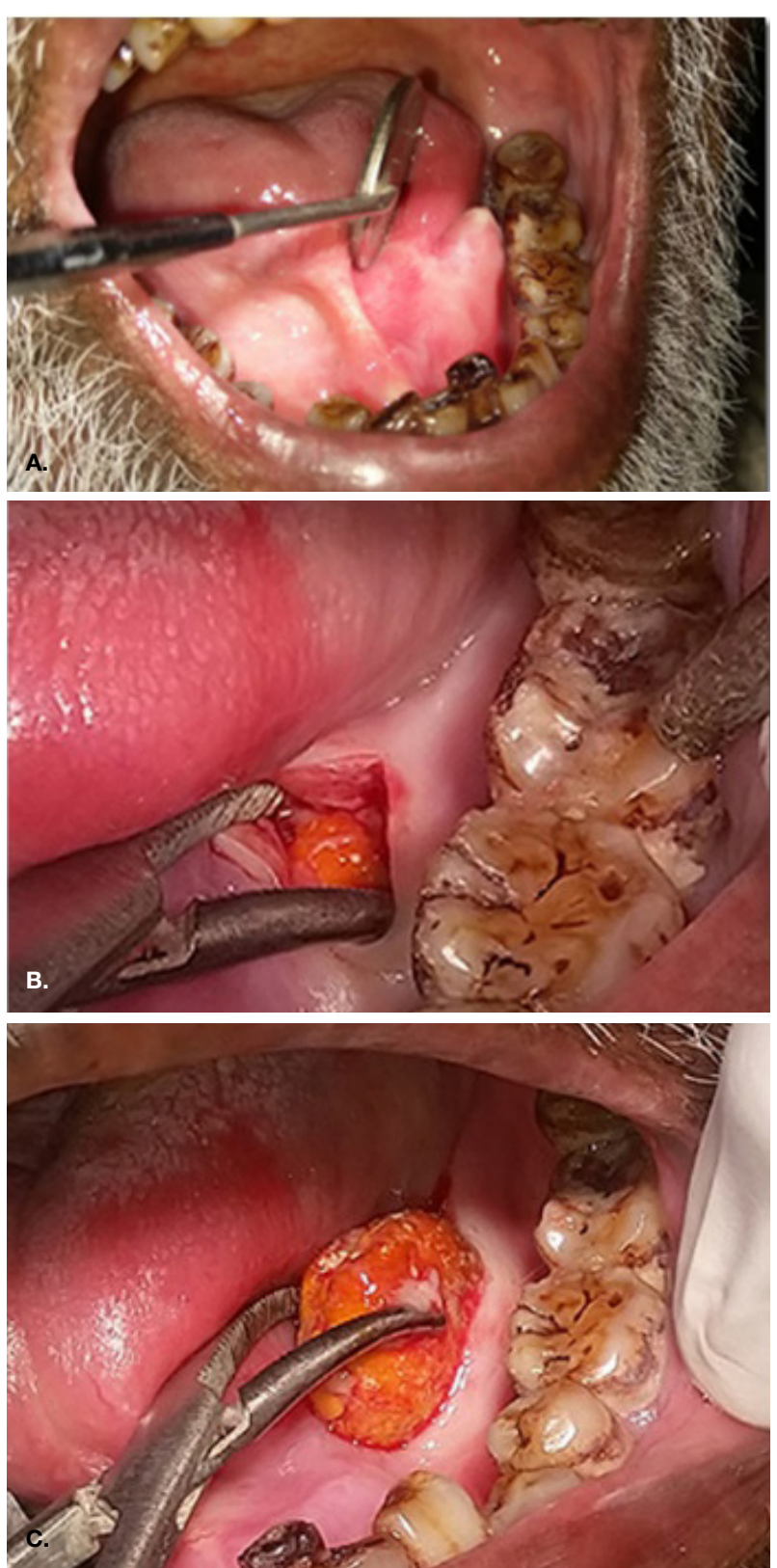

\section{Figure 1.}

a. Swelling on left floor of mouth.

b. Exposure.

c. Retrieval of sialolith from duct.

\section{DISCUSSION}

Exact cause of salivary gland calculi formation is unclear, but it is generally due to stagnation of calcium rich saliva. ${ }^{7}$ Partial obstruction of flow from the gland is more important than complete obstruction from the patient's point of view because even though complete obstruction causes stagnation of saliva, it does not cause increase in stone formation.

In completely obstruction, there is depletion of the calcium secretory granules present in the acini and this saliva is less lithogenic. ${ }^{8}$ Another theory says that for stone formation, it is likely that intermittent stasis produces a change in mucoid element of saliva, which forms a gel. This formed gel provides the framework for deposition of organic substances and salts which leads to formation of a stone. ${ }^{9}$

It also has been postulated that an unknown metabolic phenomenon can increase the salivary bicarbonate content, which alters calcium phosphate solubility and leads to precipitation of calcium and phosphorus ions. ${ }^{10}$

Irregular laminated morphology of the calculi gives an impression that it is formed intermittently. Light and electron microscopical studies have not found out any bacterial organisms or foreign bodies as the nidus for calculi formation. ${ }^{11}$

The composition is predominantly calcium phosphate and carbonate in the form of hydroxyapatite with small amounts of magnesium, potassium and ammonium, which is evenly distributed throughout the calculi. ${ }^{12}$ The organic matrix is composed of various carbohydrates and amino acids.
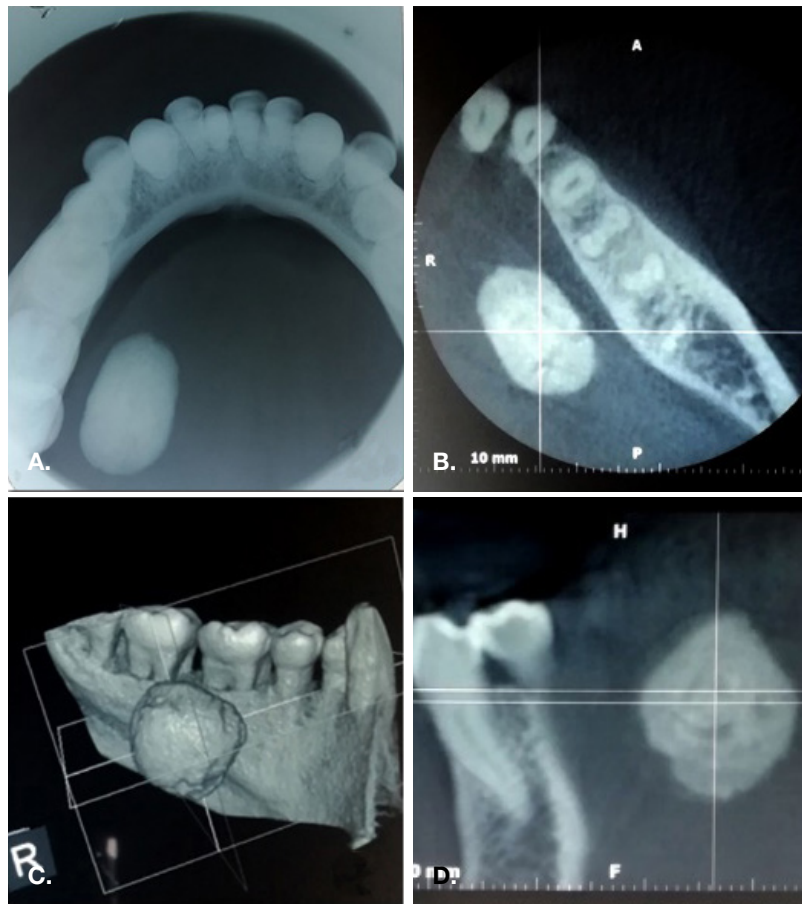

Figure 2.

a. Mandibular occlusal radiograph showing well defined oval radio-opaque structure in relation to $37-38$ region.

b. CBCT axial section showing relation of sialolith to mandible.

c. 3D Reconstruction image.

d. Transverse section showing the position of sialolith. 


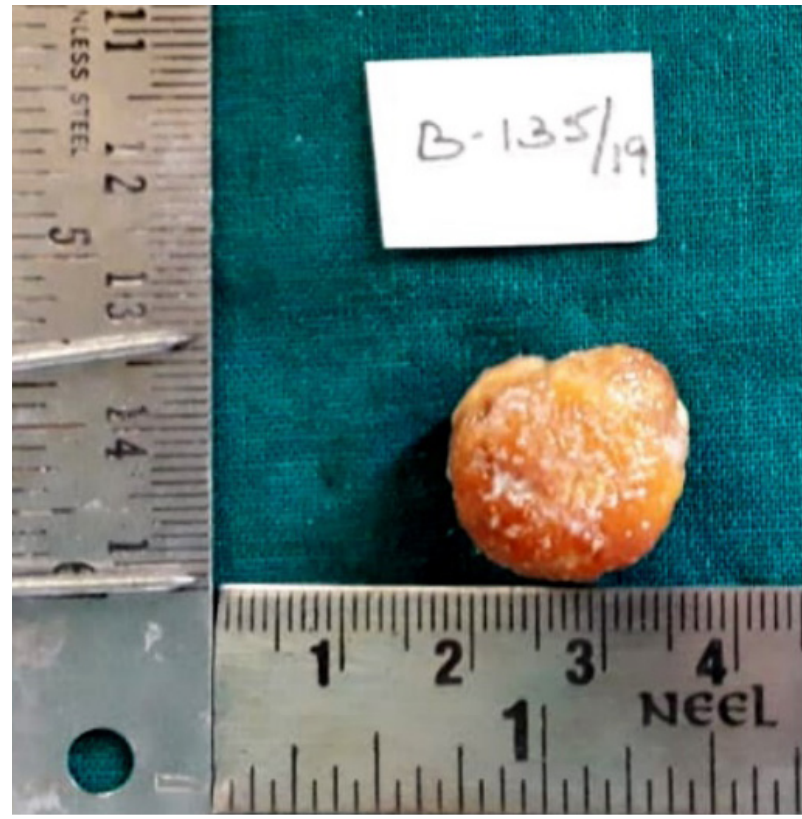

Figure 3. Sialolith after excision.

Submandibular gland stones are denser and calcium rich, related to the nature of secretions of the gland which is more mucinous compared to other glands.

The presence of a sphincter in the first $3 \mathrm{~cm}$ of the duct could result in the retrograde migration of these organic and inorganic ingredients and can lead to the formation of sialoliths, as suggested by Marchal et al. ${ }^{13}$

The factors favouring the formation of submandibular stone formation are longer and larger calibre ducts with slower salivary flow rates, salivary flow against gravity, alkalinity of the saliva secreted, and the high calcium and mucin content as mentioned earlier. ${ }^{12,14}$ Demographically it shows a male predilection. ${ }^{15}$ All ages may be affected but third to sixth decade has shown preponderance. Occurrence in the paediatric age group is rare. ${ }^{3}$

Long term obstruction without any active infection can lead to gland atrophy and ultimately leads to fibrosis and loss of secretory function. Inflammation, scarring of the duct and stricture formation are the common sequelae of intraductal stones. Strictures and stenosis lead to recurrent salivary outflow problems.

Patients presents with painful swelling in about $59 \%$ of cases whereas 29\% reported to have painless swelling. They will be afflicted with a recurrent salivary colic and spasmodic pains upon having food. They may also experience repeated infections or abscess formation. Salivary stones can also be discovered as an accidental finding on routine dental evaluation.

Careful history and examination are the key in the diagnosis of this disorder. Having the patient close the mouth slightly will aid in detection of these stones by bimanual palpation. Even the palpation of gland can be informative.

If it is found to be spongy and elastic, it suggests a healthy gland and a uniformly firm gland may be suggestive of atrophied and hypo or non-functional gland.
Plain radiography would be an excellent choice of investigation in which it be radio opaque in $80-95 \%$ of cases. Emergence of Cone Beam Computed Tomography is an easy, accurate, fast and relatively safe diagnostic tool in determining the position of the stones which can assist surgeon in planning the surgical approach. Ultrasonography is another investigation of choice to identify and locate sialoliths. It can even detect sialoliths of size $1.5 \mathrm{~mm}$ and has shown an accuracy of $99 \% .^{16}$

Submandibular sialoliths can be approached either through a transoral sialolithotomy approach or complete sialadenectomy through an extraoral approach. ${ }^{17}$ The approach basically depends on the location of stone. Sialoliths that can be palpated bimanually and localized by ultrasound within the perihilar region of the gland should be approached by transoral approach. ${ }^{18}$

The position of the submandibular gland below the mylohyoid muscle in relation to the duct which is in the floor of the mouth also favours a transoral approach to the duct rather than an extra oral approach. The risk of damage to glandular and para-glandular tissues is avoided by an intra oral approach. Gland excision should be considered in the presence of symptomatic or recurrent sialadenitis which is caused by multiple intraparenchymal stones or due to the presence of a very large stone or when minimally invasive techniques fail.

Newer techniques like sialendoscopy and laser lithotriposy are also used but these techniques have its own limitations in removing a giant sialolith. A sialolith of 4-5 $\mathrm{mm}$ can be easily removed using endoscopic technique by an experienced surgeon. ${ }^{19}$

A giant sialolith, as presented in our case report is difficult to be removed using these minimally invasive techniques, a $20 \%$ failure rate is reported by Marchal $\mathrm{F}$ even carried out by an experienced surgeon. ${ }^{20}$

Hence considering bigger size and clinically palpable sialolith without any glandular pathology we selected transoral approach for the sialolithotomy. 90\% of the total saliva is produced by the parotid, submandibular and sublingual salivary glands with $10 \%$ from minor salivary glands. The submandibular salivary gland is the largest single contributor to baseline salivary flow. After removal of the gland or duct, the patient will have severe drop-in salivary flow rates.

Glands which are severely compromised will return to normal function in $75 \%$ of cases after sialolith removal by transoral approach. Evidences exist for significant acini regeneration after duct obstruction removal. Recurrence rates in patients who have undergone transoral sialolithotomy is $18 \%$.

Lingual nerve paraesthesia is one of the common complications of the intra-oral approach. Both the lingual nerve and Wharton's duct pass deep to the mylohyoid muscle, and in the last part of the resection lingual nerve is present distal to the wharton's duct. The nerve can be identified easily and should be gently mobilised away from the surgical field preventing injury to nerve itself. 
Our patient did not experience lingual nerve paraesthesia postoperatively. An external approach carries a risk of marginal mandibular nerve injury in $0-8 \%$ of cases. ${ }^{21}$

Also, there are reported cases of a ranula developing which is formed due disruption of salivary duct. Another possible complication is sialo-cutaneous fistula due to obstruction of the ducts resulting is salivary stasis, infection and rupture through the external skin. ${ }^{22}$

The usual size of a sialolith found in Whathon's duct ranges from 1-10 $\mathrm{mm}$, in addition also there are reports of giant sialoliths greater than $3.2 \mathrm{~cm}$. Ledenma-Montes et al. found 16 cases of sialolith of size greater than $3.5 \mathrm{~mm}$ in their review of the literature. ${ }^{23}$ The largest size sialolith reported in the submandibular gland is $7.2 \mathrm{~cm}$ in length and in our case, it measured $2.2 \times 1.9 \mathrm{~cm} .{ }^{24,25}$

The mainstay of management of giant submandibular sialolith remains transoral sialolithotomy. Newer advancement is in diagnosis and localization of the calculi has helped the surgeon in planning the surgery so that the function of the salivary gland is restored with minimal or nil complications.

\section{Funding}

Nil funding received.

\section{Competing interests}

Nil conflict of interest.

\section{Ethical approval}

The manuscript was cleared by Institutional review board for this publication.

\section{Patient consent}

Consent taken from the patient.

\section{References}

1. ALMASRI, Mazen A. Management of Giant Intraglandular Submandibular Sialolith With Neck Fistula. Annals of Dentistry University of Malaya, [S.I.]. Dec 2005; 12(1): 41-5.

2. Rauch S, Gorlin RJ: Diseases of the salivary glands. In Gorlin RJ, Goldman HM (eds): Thomas' Oral Pathology, d 6. St. Louis, CV Mosby. 1970; 997-1003.

3. Bodner L, Fliss DM. Parotid and submandibular calculi in children. International journal of pediatric otorhinolaryngology. 1995 Jan 1; 31(1): 35-42.

4. Levy DM, ReMine WH, Devine KD. Salivary gland calculi: pain, swelling associated with eating. Jama. 1962 Sep 29; 181(13): 1115-9.

5. Mckenna JP, Bostock DJ, Mcmenamin PG Sialolithiasis. Am Fam Physician. 1987; 36119-125.

6. Lustmann J, Regev E, Melamed Y. Sialolithiasis: a survey on 245 patients and a review of the literature. International journal of oral and maxillofacial surgery. 1990 Jun 1; 19(3): 135-8.

7. Furstenberg A: Diseases of the salivary glands. JAMA 13614 , 1948.

8. Harrison JD, Epivatianos A, Bhatia SN. Role of microliths in the aetiology of chronic submandibular sialadenitis: a clinicopathological investigation of 154 cases. Histopathology. 1997 Sep; 31(3): 237-51.
9. Harrill JA, King Jr JS, Boyce WH. Structure and composition of salivary calculi. The Laryngoscope. 1959 May; 69(5): 48192.

10. BLATT I. Studies in sialolithiasis: III. Pathogenesis, diagnosis and treatment. Southern medical journal. 1964 Jun 1; 57(6): 723-9.

11. Brady JM, McKinney L, Stanback JS. Scanning electron microscopy of submandibular sialoliths: a preliminary report. Dentomaxillofacial Radiology. 1989 Feb; 18(1): 42-4.

12. Hiraide F, Nomura $Y$. The fine surface structure and composition of salivary calculi. The Laryngoscope. 1980 Jan; 90(1): $152-8$.

13. Marchal F, Kurt AM, Dulguerov P, Lehmann W. Retrograde theory in sialolithiasis formation. Arch Otolaryngol Head Neck. 2001; Surg 127, 66-8.

14. Raksin SZ, Gould SM, Williams AC. Submandibular duct siaIolith of unusual size and shape. J Oral Surg. 1975; 33: 142-5.

15. Auclair PL, Ellis GL, Gnepp DR, Wenig BM, Janney CG. Surgical pathology of the salivary glands. Salivary glands neoplasms: general considerations. WB Saunders, Philadelphia. 1991; 135-64.

16. Yoshimura $Y$, Inoue $Y$, Odagawa $T$. Sonographic examination of sialolithiasis. Journal of oral and maxillofacial surgery. 1989 Sep 1; 47(9): 907-12.

17. Williams MF. Sialolithiasis. Otolaryngologic Clinics of North America. 1999 Oct 1; 32(5): 819-34.

18. Zenk J, Constantinidis J, Al-Kadah B, Iro H. Transoral removal of submandibular stones. Arch Otolaryngol Head Neck Surg 2001; 127: 432-6

19. Pachisia S, Mandal G, Sahu S, Ghosh S. Submandibular sialolithiasis: A series of three case reports with review of literature. Clinics and practice. 2019 Jan 29; 9(1).

20. Marchal F. A combined endoscopic and external approach for extraction of large stones with preservation of parotid and submandibular glands. Laryngoscope 2007; 117: 373-7.

21. Ellies M, Laskawi R, Arglebe C, Schott A. Surgical management of nonneoplastic diseases of the submandibular gland: A follow-up study. International journal of oral and maxillofacial surgery. 1996 Aug 1; 25(4): 285-9.

22. Saluja H, Kasat VO, Mahindra U. Giant Sialolith in the Wharton's duct causing sialo-oral fistula: A case report and review of literature. Journal of Orofacial Sciences. 2012 Jul 1; 4(2): 137

23. Ledesma-Montes C, Garcés-Ortíz M, Salcido-García JF, Hernández-Flores F, Hernández-Guerrero JC. Giant sialolith: case report and review of the literature. Journal of oral and maxillofacial surgery. 2007 Jan 1; 65(1): 128-30.

24. Vittal U, Shetty SC, Manvikar P, Kini U, Gupta S. Giant Sialolith (megalith) of submandibular salivary gland. Australian Journal of Oto-Laryngology. 2002 Apr 1; 5(1): 43.

25. Rai M, Burman R. Giant submandibular sialolith of remarkable size in the comma area of wharton's duct: A case report. J Oral Maxillofac Surg. 2009; 67: 1329-32 